\title{
Correction: Using an Electronic App to Promote Home-Based Self-Care in Older Patients With Heart Failure: Qualitative Study on Patient and Informal Caregiver Challenges
}

Sahr Wali ${ }^{1,2}$, MSc; Karim Keshavjee ${ }^{2,3}$, MSc, MBA, MD, CCFP; Linda Nguyen ${ }^{4}$, MSc; Lawrence Mbuagbaw ${ }^{5}$, MD, $^{2}$ MPH, PhD, FRSPH; Catherine Demers ${ }^{5,6}$, MD, MSc, FRCPC

${ }^{1}$ Centre for Global eHealth Innovation, Techna Institute, University Health Network, Toronto, ON, Canada

${ }^{2}$ Institute of Health Policy, Management and Evaluation, Dalla Lana School of Public Health, University of Toronto, Toronto, ON, Canada

${ }^{3}$ InfoClin, Toronto, ON, Canada

${ }^{4}$ School of Rehabilitation Science, Faculty of Health Sciences, McMaster University, Hamilton, ON, Canada

${ }^{5}$ Department of Health Research Methods, Evidence and Impact, McMaster University, Hamilton, ON, Canada

${ }^{6}$ Department of Medicine, McMaster University, Hamilton, ON, Canada

\section{Corresponding Author:}

Catherine Demers, MD, MSc, FRCPC

Department of Health Research Methods, Evidence and Impact

McMaster University

237 Barton St E

Hamilton, ON, L8L 2X2

Canada

Phone: 19055259140 ext 73324

Email: demers@hhsc.ca

\section{Related Article:}

Correction of: https://cardio.jmir.org/2020/1/e15885/

(JMIR Cardio 2020;4(1):e25624) doi: 10.2196/25624

In "Using an Electronic App to Promote Home-Based Self-Care in Older Patients With Heart Failure: Qualitative Study on Patient and Informal Caregiver Challenges" (JMIR Cardio 2020;4(1):e15885) the authors noted two errors.

In the originally published article, Sahr Wali was listed as the Corresponding Author. The Corresponding Author address was listed as:

Institute of Health Policy, Management and
Evaluation
Dalla Lana School of Public Health
University of Toronto
155 College St
Toronto, ON, M5T3M6
Canada
Phone: 14169784326
Email: sahrwali@mail.utoronto.ca

In the corrected version, the Corresponding Author has been changed to Catherine Demers, with the Corresponding Author address:
Department of Health Research Methods, Evidence and Impact

McMaster University

237 Barton St $E$

Hamilton, ON, L8L $2 X 2$

Canada

Phone: 19055259140 ext 73324

Email:demers@hhsc.ca

An Acknowledgements section has also been added to the original paper with the following text:

This work was completed at McMaster University.

The correction will appear in the online version of the paper on the JMIR Publications website on November 11, 2020, together with the publication of this correction notice. Because this was made after submission to PubMed, PubMed Central, and other full-text repositories, the corrected article has also been resubmitted to those repositories. 
This is a non-peer-reviewed article. Submitted 09.11.20; accepted 10.11.20; published 11.11.20.

Please cite as:

Wali S, Keshavjee K, Nguyen L, Mbuagbaw L, Demers C

Correction: Using an Electronic App to Promote Home-Based Self-Care in Older Patients With Heart Failure: Qualitative Study on

Patient and Informal Caregiver Challenges

JMIR Cardio 2020;4(1):e25624

URL: http://cardio.jmir.org/2020/1/e25624/

doi: $10.2196 / 25624$

PMID: $\underline{3175695}$

(CSahr Wali, Karim Keshavjee, Linda Nguyen, Lawrence Mbuagbaw, Catherine Demers. Originally published in JMIR Cardio (http://cardio.jmir.org), 11.11.2020. This is an open-access article distributed under the terms of the Creative Commons Attribution License (https://creativecommons.org/licenses/by/4.0/), which permits unrestricted use, distribution, and reproduction in any medium, provided the original work, first published in JMIR Cardio, is properly cited. The complete bibliographic information, a link to the original publication on http://cardio.jmir.org, as well as this copyright and license information must be included. 